\title{
Los cursos múltiples de corticoides en la amenaza de parto prematuro no mejoran los resultados neonatales y afectan el crecimiento
}

Multiple courses of antenatal corticosteroids for preterm birth does not improve neonatal outcomes and affect growing

Murphy K y col. Lancet 2008; 372:2143-51

\section{Objetivo}

Evaluar si los cursos múltiples de corticoides durante el embarazo reducen la morbi-mortalidad neonatal y/o afectan el crecimiento fetal.

\section{Diseño, lugar y pacientes}

Ensayo clínico aleatorizado doble ciego con cinco años de seguimiento realizado en 80 centros de 20 países. Fueron aleatorizadas 1858 embarazadas (451 de Argentina) de 25 a 32 semanas de gestación que permanecían en riesgo de parto prematuro después de 14 a 21 días de haber recibido el primer curso de corticoides.

\section{Intervención y medición de resultados principales}

Dos dosis de $12 \mathrm{mg}$ betametasona o placebo (separadas por 24 horas) cada 14 días y hasta la semana 33 o hasta el parto (lo que ocurriera antes). El resultado principal fue un resultado compuesto de muerte perinatal o neonatal y morbilidad neonatal; esta última definida por síndrome de distrés respiratorio severo, hemorragia intraventricular grado III o IV, leucomalacia periventricular, displasia broncopulmonar o enterocolitis necrotizante.

\section{Resultados principales}

La morbimortalidad neonatal fue similar entre los expuestos a cursos múltiples de corticoides y los expuestos a placebo (tabla 1).
Tabla 1: comparación de los resultados neonatales entre los cursos múltiples de corticoides y el placebo.

\begin{tabular}{l|c|c|c}
\multicolumn{1}{c|}{ Resultados } & Corticoides & Placebo & p \\
\hline Morbimortaldad neonatal & $12,9 \%$ & $12,5 \%$ & 0,83 \\
\hline Peso & $2216 \mathrm{~g}$ & $2330 \mathrm{~g}$ & 0,0026 \\
\hline Talla & $44,5 \mathrm{~cm}$ & $45,4 \mathrm{~cm}$ & $<0,001$ \\
\hline Circunferencia cefálica & $31,1 \mathrm{~cm}$ & $31,7 \mathrm{~cm}$ & $<0,001$ \\
\hline
\end{tabular}

Se descartaron interacciones ${ }^{*}$ con la edad gestacional al ingreso, rotura prematura de membranas, y gestación múltiple. No hubo diferencias en la morbilidad infectológica materna.

\section{Conclusiones}

Los cursos múltiples de corticoesteroides prenatales cada 14 días, no mejoran los resultados neonatales y se asocian con una disminución del peso, la talla y la circunferencia cefálica, por lo que no se recomienda dicha intervención.

Palabras claves: corticoides, cuidados prenatales, peso, talla, resultados neonatales. Keywords: steroids, prenatal care, weight, length, neonatal outcome. Fuentes de financiamiento: Canadian Institutes of Health Research.

\section{Comentario}

Desde que Liggins ${ }^{1}$ observó en la década del 1960 que los corticoides prenatales mejoraban la función pulmonar, más de 4000 mujeres y sus hijos han sido incluidos en estudios aleatorizados, demostrándose los beneficios sobre la mortalidad y morbilidad neonatal de los cursos únicos de corticoides.

Por otro lado, estudios de laboratorio mostraron que el efecto de los corticoides disminuía luego de siete días, por lo que surgió la idea de repetir los cursos de corticoides. Sin embargo, posteriormente, estudios en animales y observacionales, sugirieron que las dosis múltiples de corticoides podrían asociarse a trastornos del crecimiento somático y visceral, así como a retraso en la mielinización.

Previamente al trabajo de Murphy y col. (MACS) que hemos resumido, una revisión Cochrane ${ }^{2}$ que había incluido los resultados de cuatro estudios informó que los cursos semanales de corticoides se asociaban a una menor incidencia (RR: 0,82; IC95\% 0,72 a 0,93) y severidad (RR: 0,60; IC95\% 0,48 a 0,75) de enfermedad respiratoria neonatal.

Sin embargo, el que hoy nos ocupa es el ensayo más grande de cursos múltiples publicado hasta la fecha y contempla el seguimiento de los niños hasta los cinco años de edad, con el foco puesto en la evaluación de su crecimiento, conducta, tolerancia a la glucosa y presión arterial. En concordancia con el estudio del $\mathrm{NICHD}^{3}$, los niños que recibieron dosis múltiples evidenciaron menor peso, talla y circunferencia cefálica. Aún mayor preocupación generó el hecho de que en el ensayo del $\mathrm{NICHD}^{3}$, y durante el seguimiento a dos años, hubo una mayor incidencia de parálisis cerebral en los mayores de 34 semanas del grupo expuesto. Aunque la relevancia clínica de estos hallazgos aún se desconoce, el MACS seguramente proveerá algunas respuestas cuando se complete, con la ventaja de que sus resultados son extrapolables a nuestra población (cerca del $25 \%$ de las participantes).

\section{Conclusiones del comentador}

Mientras no se cuente con los datos del seguimiento a largo plazo de estos niños es recomendable no utilizar cursos múltiples de corticoides.

Ver glosario*

Gustavo Izbizky [ Sección Medicina Fetal y Genética, Servicio de Obstetricia, Hospital Italiano de Buenos Aires. gustavo.izbizky@hospitalitaliano.org.ar ]

Izbizky G. Los cursos múltiples de corticoides en la amenaza de parto prematuro no mejoran los resultados neonatales y afectan el crecimiento. Evid. actual. práct. ambul; 12(1): 5, Ene-Mar.2009. Murphy $\mathrm{K}$ y col. Comentado de: Multiple courses of antenatal corticosteroids for preterm birth (MACS): a randomised controlled trial. Lancet 2008; 372: 2143-51.

PMID: 19101390. Disponible en bajo suscripción

URL: http://www.thelancet.com/journals/lancet/article/PIIS0140-6736(08)61929-7/fulltext\#article_upsell (último acceso 14/02/09).

\section{Referencia}

1. Liggins G y col. A controlled trial of antepartum glucocorticoid treatment for prevention of the respiratory distress syndrome in premature infants. Pediatrics $1972 ; 50: 515-25$ 2. Crowther $\mathrm{C}$ y col. Repeat doses of prenatal corticosteroids forwomen at risk of pretermbirth for preventing neonatal respiratory disease. Cochrane Database of Systematic Reviews 2007, Issue 3. Art. No.: CD003935. DOI: 10.1002/14651858.CD003935

3. Wapner R y col. for the National Institute of Child Health and Human Development Maternal-Fetal Medicine Units Network Long-Term Outcomes after Repeat Doses of Antenatal Corticosteroids. N Engl J Med 2007;357:1190-8. 